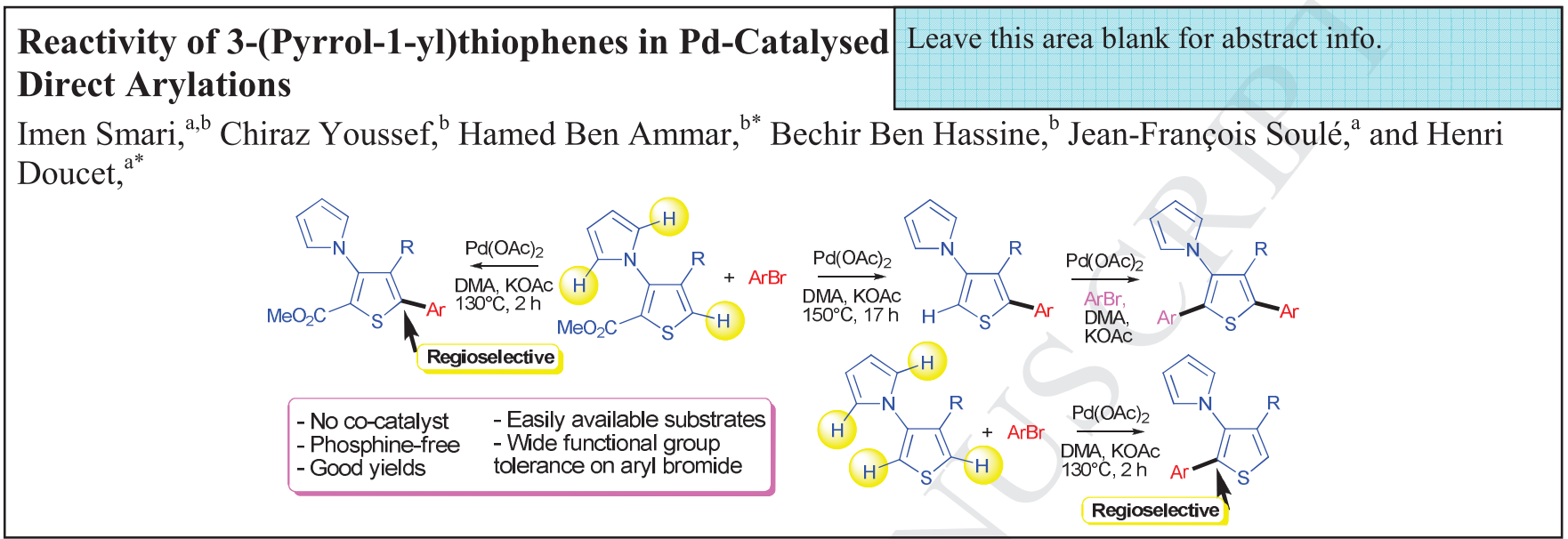




\title{
Reactivity of 3-(Pyrrol-1-yl)thiophenes in Pd-Catalysed Direct Arylations
}

\author{
Imen Smari, ${ }^{\mathrm{a}, \mathrm{b}}$ Chiraz Youssef, ${ }^{\mathrm{b}}$ Hamed Ben Ammar, ${ }^{\mathrm{b}}$ Bechir Ben Hassine, ${ }^{\mathrm{b}}$ Jean-François \\ Soulé, ${ }^{\mathrm{a}}$ and Henri Doucet, ${ }^{\mathrm{a}^{*}}$ \\ ${ }^{a}$ Institut des Sciences Chimiques de Rennes, UMR 6226 CNRS-Université de Rennes \\ "Organométalliques: Matériaux et Catalyse", Campus de Beaulieu, 35042 Rennes, France. \\ ${ }^{b}$ Laboratoire de Synthèse Organique Asymétrique et Catalyse Homogène, (UR 11ES56) Université de Monastir, Faculté des \\ Sciences de Monastir, avenue de l'environnement, Monastir 5000, Tunisie. Fax: (+216) 73 500 278; Tel: (+216) 73 500 275.

\begin{abstract}
The regioselectivity of the Pd-catalysed direct arylation of 3-(pyrrol-1-yl)thiophene derivatives was investigated. Conditions allowing either the regioselective arylation at $\mathrm{C} 2$ or at $\mathrm{C} 5$ of the thiophene ring are reported. From methyl 3-(pyrrol-1-yl)thiophene-2carboxylate using KOAc as the base, DMA as the solvent and only $1 \mathrm{~mol} \% \mathrm{Pd}(\mathrm{OAc})_{2}$ catalyst, the target 5-arylated thiophenes were obtained in moderate to good yields with a wide variety of aryl halides; whereas the use of 1-(4-methylthiophen-3-yl)-pyrrole affords the $\mathrm{C} 2$-arylated thiophenes. The sequential palladium catalysed 2,5-diheteroarylation of such 3-(pyrrol-1-yl)thiophene is also reported allowing the access to thiophenes bearing two different aryl units at $\mathrm{C} 2$ and $\mathrm{C} 5$. A pyrazole bearing an ester substituent at C4 and a pyrrole
\end{abstract} \\ substituent at $\mathrm{C} 5$ was only arylated at $\mathrm{C} 2$ on the pyrrole ring.
}

\section{Introduction}

Both thiophene and pyrrole derivatives represent important structures due to their biological and/or physical properties. For example, Motapizone ${ }^{1 \mathrm{a}}$ is used against platelet aggregation, Saviprazole ${ }^{1 b}$ is a gastric proton pump inhibitor and Tiflucarbine ${ }^{1 \mathrm{c}}$ displays antidepressant properties (Fig. 1). Due to these properties, the discovery of simple accesses to a variety of thiophene or pyrrole derivatives remains an important challenge for organic chemists.

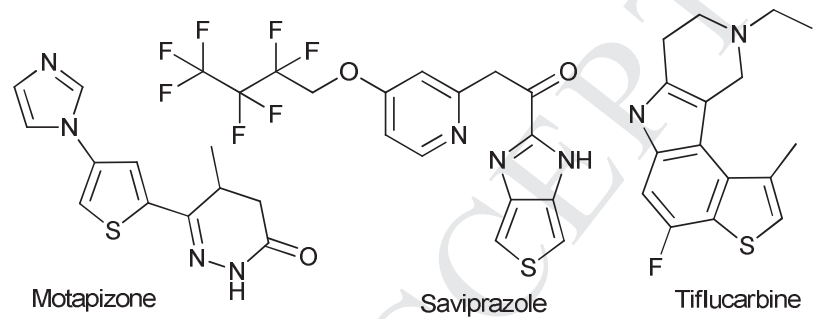

Figure 1. Examples of bioactive thiophene derivatives

As early as in 1985-1992, Ohta et al. reported the direct arylation of heteroaromatics with aryl halides via a $\mathrm{C}-\mathrm{H}$ bond activation using $\mathrm{Pd}\left(\mathrm{PPh}_{3}\right)_{4}$ catalyst. ${ }^{2}$ Since these results, the $\mathrm{Pd}$-catalysed direct arylation of several heteroaryls using aryl halides as the coupling partners has proved to be a very powerful method for a simpler and greener access to a wide variety of arylated heterocycles. ${ }^{3,4}$ This method is very attractive as it avoids the preparation of an organometallic derivative and as the major by-products of the reaction are a base associated to HX, instead of metallic salts. ${ }^{5}$

The direct arylation of a variety of thiophene and pyrrole derivatives has been reported in recent years. ${ }^{6,7}$ However, to our knowledge, the reactivity of a thiophene substituted by a pyrrole unit at C3 and also of a 5-pyrrolylpyrazole for Pd-catalysed direct arylations has not been described Therefore, the reactivity for direct arylation of such substrates needed to be investigated.

Here, we wish to report (i) conditions allowing a regioselective arylation of a 3-(pyrrol-1-yl)thiophene derivative, (ii) a one pot procedure with arylation at $\mathrm{C} 5$ of the thiophene derivative followed by decarboxylation at $\mathrm{C} 2$ and (iii) the sequential arylation to prepare 2,5diarylated thiophenes with two different aryl units.

\section{Results and discussion}

Based on previous results, for this study DMA was initially chosen as the solvent and KOAc as the base. ${ }^{8}$ The reactions were performed at $130-150{ }^{\circ} \mathrm{C}$ under argon in the presence of $\mathrm{Pd}(\mathrm{OAc})_{2}$ or $\mathrm{PdCl}\left(\mathrm{C}_{3} \mathrm{H}_{5}\right)(\mathrm{dppb})$ catalysts. First, using only $1 \mathrm{~mol} \% \mathrm{Pd}(\mathrm{OAc})_{2}$, the reaction of 1 equiv. of 3-bromonitrobenzene with 1.5 equiv. of methyl 4-methyl-3-(pyrrol-1-yl)thiophene-2carboxylate as the coupling partners at $150{ }^{\circ} \mathrm{C}$ during $17 \mathrm{~h}$ affords selectively the in situ decarboxylated C5-arylation product $\mathbf{1 b}$ in $78 \%$ yield (Table 1 , entry 1 ). On the other hand, the reaction performed under the same reaction conditions, but after only $2 \mathrm{~h}$ affords a mixture of $\mathbf{1 a}$ and $\mathbf{1 b}$ in a 83:17 ratio (Table 1 , entry 2 ).

\footnotetext{
${ }^{*}$ Corresponding author. Tel.: 00-33-2-23-23-63-84; fax: 00-33-2-23-23-69-39; e-mail: henri.doucet@univ-rennes1.fr
} 

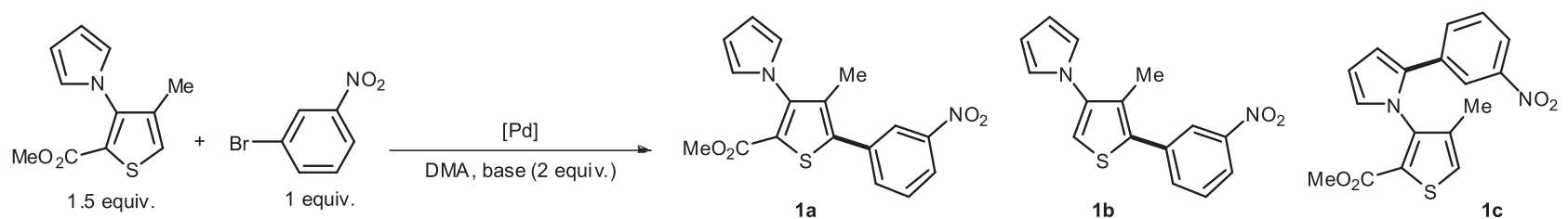

Scheme 2. Regioselectivity of the palladium catalysed direct arylation using methyl 4-methyl-3-(pyrrol-1-yl)thiophene-2carboxylate and 3-bromonitrobenzene as the coupling partners

Table 1. Influence of the reaction conditions for the palladium catalysed direct arylation using methyl 4-methyl-3-(pyrrol-1yl)thiophene-2-carboxylate and 3-bromonitrobenzene as the coupling partners (Scheme 2)

\begin{tabular}{|c|c|c|c|c|c|c|}
\hline Entry & Catalyst (mol\%) & Base & Time (h) & Temp $\left({ }^{\circ} \mathrm{C}\right)$ & Ratio 1a:1b:1c & Yield in $\mathbf{1 a}$ or $\mathbf{1 b}(\%)$ \\
\hline 1 & $\mathrm{Pd}(\mathrm{OAc})_{2}(1)$ & KOAc & 17 & 150 & $0: 100: 0$ & 76 of $\mathbf{1 b}$ \\
\hline 2 & $\mathrm{Pd}(\mathrm{OAc})_{2}(1)$ & KOAc & 2 & 150 & $83: 17: 0$ & - \\
\hline 3 & $\mathrm{Pd}(\mathrm{OAc})_{2}(1)$ & KOAc & 2 & 130 & $93: 7: 0$ & 78 of $1 a$ \\
\hline 4 & $\mathrm{Pd}(\mathrm{OAc})_{2}(1)$ & $\mathrm{CsOAc}$ & 17 & 150 & $49: 51: 0$ & - \\
\hline 5 & $\mathrm{Pd}(\mathrm{OAc})_{2}(1)$ & $\mathrm{CsOAc}$ & 2 & 130 & $94: 6: 0$ & 78 of $1 \mathbf{a}$ \\
\hline 6 & $\mathrm{Pd}(\mathrm{OAc})_{2}(1)$ & $\mathrm{NaOAc}$ & 17 & 150 & $23: 77: 0$ & - \\
\hline 7 & $\mathrm{Pd}(\mathrm{OAc})_{2}(1)$ & $\mathrm{NaOAc}$ & 2 & 130 & $69: 31: 0$ & - \\
\hline 8 & $\mathrm{Pd}(\mathrm{OAc})_{2}(1)$ & $\mathrm{Cs}_{2} \mathrm{CO}_{3}$ & 17 & 150 & 0:100:0 & 77 of $\mathbf{1 b}$ \\
\hline 9 & $\mathrm{PdCl}\left(\mathrm{C}_{3} \mathrm{H}_{5}\right)(\mathrm{dppb})(2)$ & KOAc & 17 & 150 & $14: 86: 0$ & 70 of $\mathbf{1 b}$ \\
\hline
\end{tabular}

Conditions: 3-bromonitrobenzene (1 equiv.), 4-methyl-3-(pyrrol-1-yl)thiophene-2-carboxylate (1.5 equiv.), base (2 equiv.), DMA, isolated yields, complete conversion of 3-bromonitrobenzene was observed in all cases.

It should be noted that in both cases no arylation at the pyrrole ring to afford 1c was observed by GC/MS analysis of the crude mixture. A lower temperature led to an increase in the selectivity for $\mathbf{1 a}$, as at $130^{\circ} \mathrm{C}$ ratio $\mathbf{1 a}: \mathbf{1 b}$ was $93: 7$ and 1a was isolated in $78 \%$ yield (Table 1 , entry 3 ). Then, we examined the influence of the nature of the base for this reaction. The use of CsOAc instead of KOAc at $150{ }^{\circ} \mathrm{C}$ afforded lower amounts of decarboxylated product $\mathbf{1 b}$, and at $130{ }^{\circ} \mathrm{C}$ a $94: 6$ ratio (1a:1b) was obtained, with $78 \%$ isolated yield of $\mathbf{1 a}$ (Table 1 , entries 4 and 5). NaOAc led to mixtures of products $\mathbf{1 a}$ and $\mathbf{1 b}$, whereas $\mathrm{Cs}_{2} \mathrm{CO}_{3}$ afforded exclusively $\mathbf{1 b}$ (Table 1 , entries 6-8). The use of $2 \mathrm{~mol} \% \mathrm{PdCl}\left(\mathrm{C}_{3} \mathrm{H}_{5}\right)(\mathrm{dppb})$ catalyst $^{8 \mathrm{~b}}$ instead of $1 \mathrm{~mol} \% \mathrm{Pd}(\mathrm{OAc})_{2}$, also affords $\mathbf{1 b}$ although in slightly lower yield (Table 1 , entry 9 ).

Then, using the most effective reaction conditions for coupling with and without decarboxylation (DMA, KOAc, $\mathrm{Pd}(\mathrm{OAc})_{2}, 130{ }^{\circ} \mathrm{C} 2 \mathrm{~h}$ or $150{ }^{\circ} \mathrm{C} 17 \mathrm{~h}$ ), we explored the scope of this reaction using a variety of aryl bromides as the coupling partner (Schemes 3 and 4).

First, we investigated the reaction of methyl 4-methyl-3(pyrrol-1-yl)thiophene-2-carboxylate using $1 \mathrm{~mol} \%$ $\mathrm{Pd}(\mathrm{OAc})_{2}$ catalyst with a set of aryl bromides at $130{ }^{\circ} \mathrm{C}$ during $2 \mathrm{~h}$ in order to obtain the non-decarboxylated products (Scheme 3). The reaction with the parasubstituted aryl bromides, 4-bromobenzonitrile, 4bromopropiophenone or 4-bromoanisole proceeds very smoothly to afford the target products $\mathbf{2 - 5}$ in $75-83 \%$ yields. Meta-substituted 3-bromoacetophenone and also 4-bromo-1-nitro-2-(trifluoromethyl)benzene also react nicely to give $\mathbf{6}$ and 7 in $79 \%$ and $60 \%$ yields, respectively. 3-Bromopyridine, 5-bromonicotinonitrile and 5-bromopyrimidine were also successfully coupled with methyl 4-methyl-3-(pyrrol-1-yl)thiophene-2carboxylate, without decarboxylation, to afford 8-10 in 58$79 \%$ yields. 

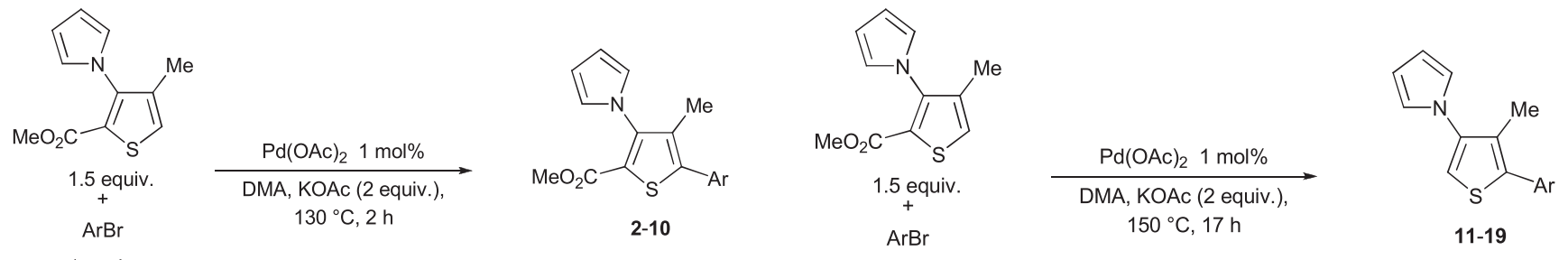
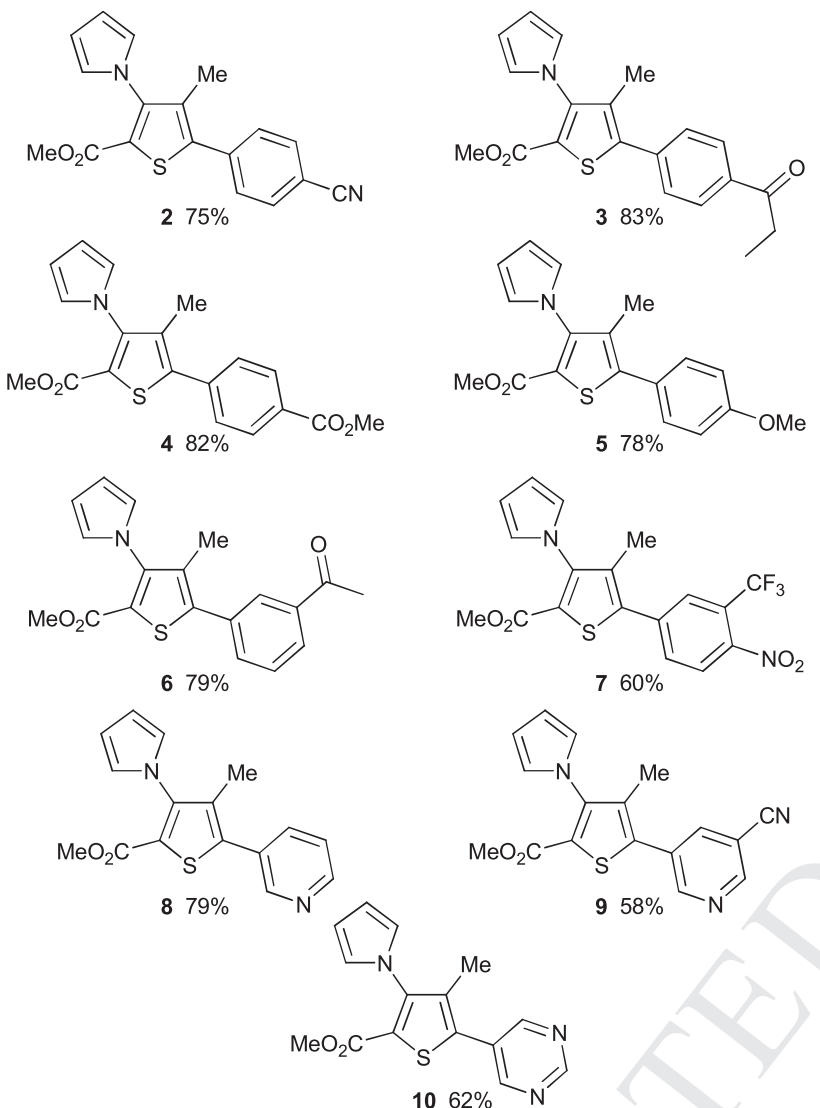

$062 \% \quad N$

Scheme 3. Scope of the C5-arylation of methyl 4-methyl3-(pyrrol-1-yl)thiophene-2-carboxylate

The scope of the C5-arylation with in situ decarboxylation was also studied (Scheme 4). From 4-cyano-, 4propionyl- and 4-chlorobromobenzene, after $17 \mathrm{~h}$ at 150 ${ }^{\circ} \mathrm{C}$, the decarboxylated C5-arylated thiophenes 11-13 were obtained in good yields. On the other hand, no formation of the desired decarboxylated product 14 was observed in the presence of the electron-rich 4-bromoanisole as only product 5 was formed (68\%). The decarboxylation step appears to be favoured by the presence of electronwithdrawing substituents on the arene. In the presence of 2-bromobenzonitrile and 4-bromo-1-nitro-2(trifluoromethyl)benzene, $\mathbf{1 5}$ and $\mathbf{1 6}$ were obtained in 79\% and $70 \%$ yields, respectively. Then, three heteroarenes were employed. 3-Bromopyridine, 5-bromonicotinonitrile and 5-bromopyrimidine also afforded the desired decarboxylated products $\mathbf{1 7 - 1 9}$ in good yields.

1 equiv.

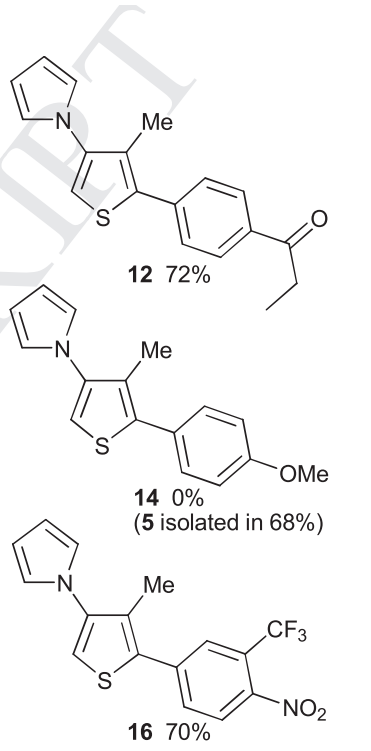<smiles>Cc1c(-n2cccc2)csc1-c1cnc2ccccc2c1</smiles>

$1761 \%$<smiles>Cc1c(-n2cccc2)csc1-c1cncnc1</smiles>

Scheme 4. Scope of the C5-arylation of methyl 4-methyl3-(pyrrol-1-yl)thiophene-2-carboxylate with in-situ decarboxylation

The selectivity of the arylation of 3-(pyrrol-1-yl)thiophene derivatives appears to be very sensitive to the steric hindrance of the pyrrolyl moiety. The presence of a substituent at $\mathrm{C} 4$ on the thienyl ring seems to be crucial to control the selectivity. Indeed, in the presence of methyl 3-(pyrrol-1-yl)thiophene-2-carboxylate and 4bromobenzonitrile as coupling partner, the formation of a mixture of the 5-arylated thiophene 20a and 2-arylated pyrrole 20b in a 31:35 ratio was observed under the same reaction conditions (Scheme 5). 


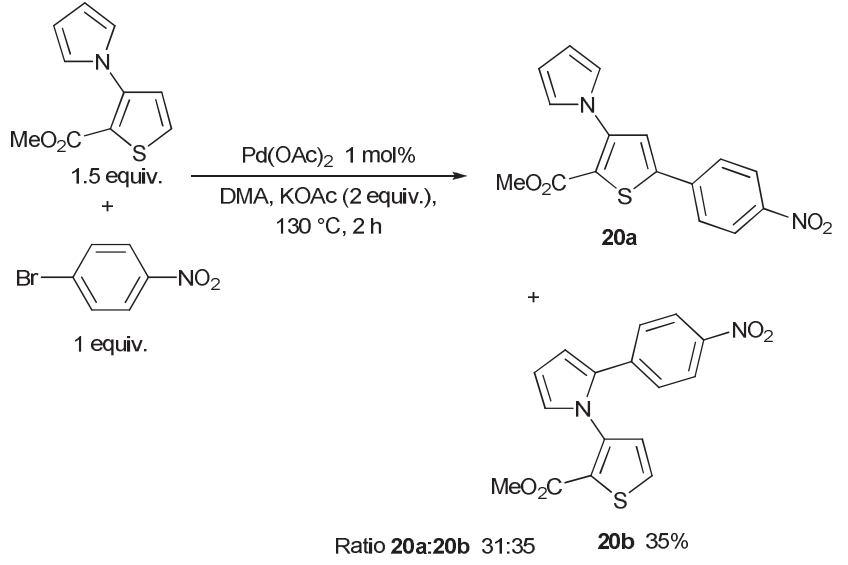

Scheme 5. Regioselectivity of the arylation of methyl 3(pyrrol-1-yl)thiophene-2-carboxylate

The regioselectivity of the arylation of 1-(4methylthiophen-3-yl)-pyrrole was also investigated (Scheme 6). In the presence of 4-bromobenzonitrile and 1 $\mathrm{mol} \% \mathrm{Pd}(\mathrm{OAc})_{2}$ catalyst, the regiospecific formation of the $\mathrm{C} 2$-arylated product $\mathbf{2 1}$ was observed which indicates that the most reactive position of a 3-pyrrolylthiophene is the carbon $\mathrm{C} 2$ of the thienyl ring. Therefore, this result confirms that an ester substituent at $\mathrm{C} 2$ on the thienyl ring acts as a blocking group, allowing for selective arylation at C5 position. A similar regioselectivity trend for direct arylations had been previously observed using 3aminothiophene derivatives. ${ }^{9}$
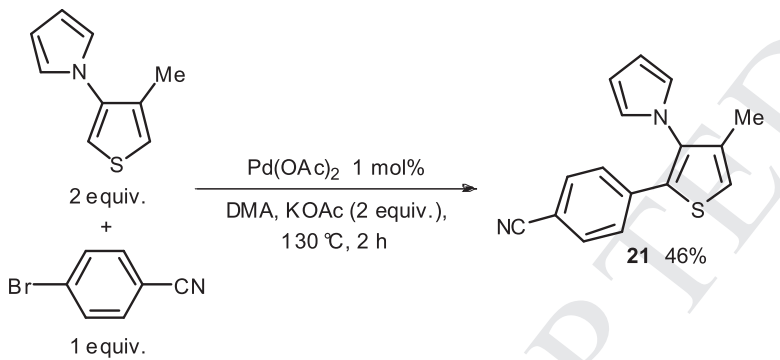

Scheme 6. Regioselectivity of the arylation of 1-(4methylthiophen-3-yl)-pyrrole

The reactivity of the arylated thiophene derivatives $\mathbf{1 2}$ and 17 for C5 arylation was then evaluated (Scheme 7). Such arylations would allow the preparation of nonsymmetrically 2,5-diarylated thiophene derivatives. From 17 and bromobenzene the desired product 22 was obtained in 56\% yield. 12 reacted with 5-bromopyridine also affords the desired 2,5-diarylated thiophene $\mathbf{2 3}$ in good yield.

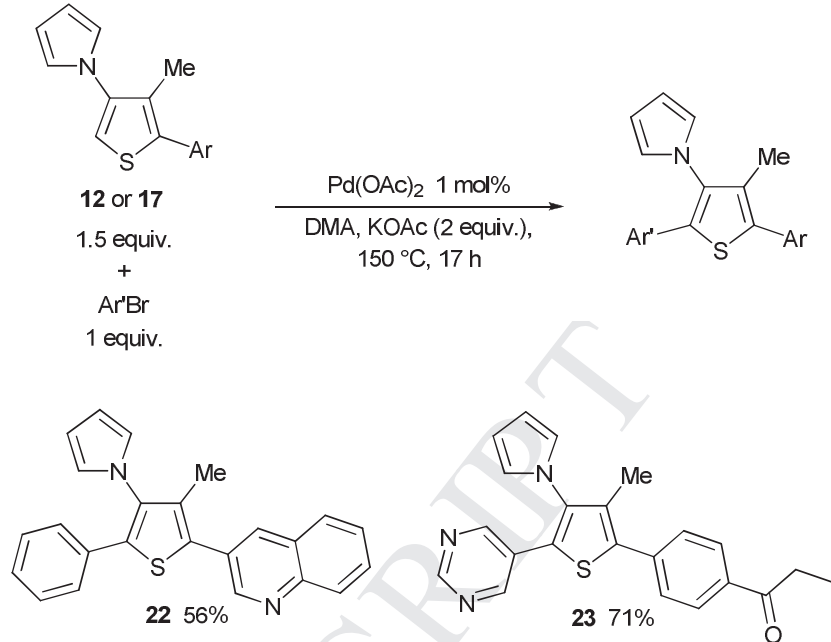

Scheme 7. C5-arylation of 2-aryl-3-methyl-4-(pyrrol-1yl)thiophenes

The reactivity of 2-(pyrrol-1-yl)thiophene-3-carbonitrile in the presence of 3-bromonitrobenzene was also investigated (Scheme 8). A permutation of the electronwithdrawing (pyrrolyl) and electron-donating (ester or cyano) substituents on the thiophene was found to drastically modify the reactivity. In the presence of 1 $\mathrm{mol} \% \mathrm{Pd}(\mathrm{OAc})_{2}$ catalyst and KOAc as base, the expected product 24a was obtained in a very low yield and the $\mathrm{C} 2$ arylated pyrrole $\mathbf{2 4 b}$ was not observed.

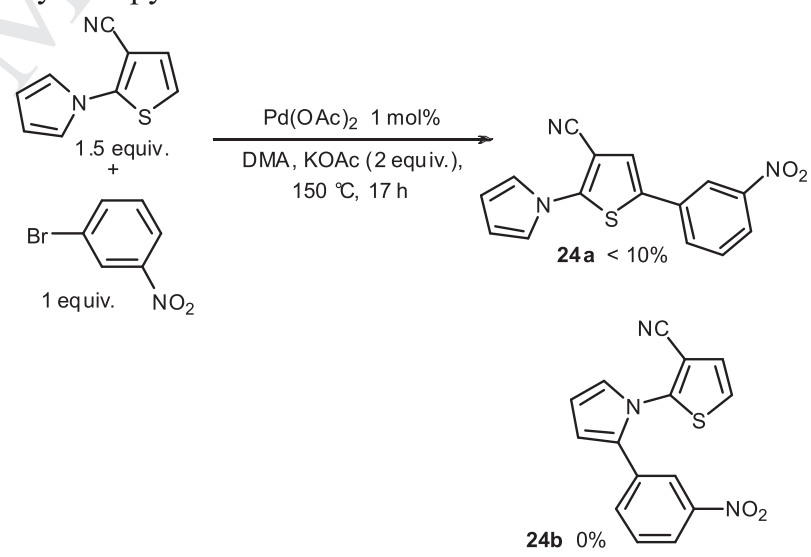

Scheme 8. Reactivity of 2-(pyrrol-1-yl)thiophene-3carbonitrile

We also explored the reactivity of a pyrazole bearing a pyrrole substituent at C5 and an ester at C4 (Scheme 9). As the position $\mathrm{C} 3$ of pyrazoles derivatives generally exhibits a poor reactivity, the formation of product $\mathbf{2 5 b}$ was expected. Indeed, 4-bromobenzonitrile and this 5(pyrrol-1-yl)-pyrazole in the presence of $1 \mathrm{~mol} \%$ $\mathrm{Pd}(\mathrm{OAc})_{2}$ and $\mathrm{KOAc}$ in DMA at $130{ }^{\circ} \mathrm{C}, 2$ or $17 \mathrm{~h}$ affords the $\mathrm{C} 2$-arylated pyrrole $\mathbf{2 5 b}$ in $42 \%$ yield. No formation of 25a was observed. A similar regioselectivity was observed in the presence of 4-bromonitrobenzene, 4bromobenzaldehyde, 4-bromo-1-nitro-2(trifluoromethyl)benzene and 5-bromopyrimidine as the coupling partners, and products 26-29 were obtained in 
$40-58 \%$ yields, respectively. The formation of diarylation products in trace amounts was also observed by GC/MS analysis of the crude mixtures.

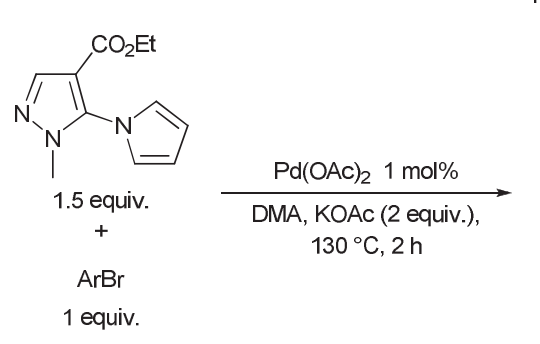

$2640 \%$<smiles>CCOC(=O)c1cnn(C)c1-n1cccc1-c1ccc(C=O)cc1</smiles>

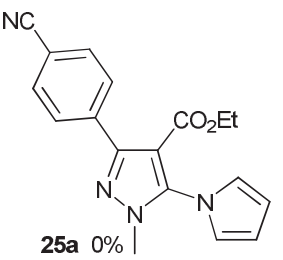

25a $0 \%$

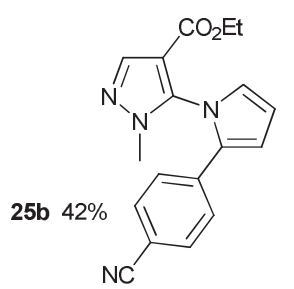

Scheme 9. Arylation of ethyl 1-methyl-5-(pyrrol-1-yl)pyrazole-4-carboxylate

In conclusion, thiophenes bearing a pyrrole unit at $\mathrm{C} 3$ and a methyl at $\mathrm{C} 4$ were found to react exclusively at the thienyl ring. We established that $1 \mathrm{~mol} \%$ of air stable palladium $\mathrm{Pd}(\mathrm{OAc})_{2}$ catalyst and $\mathrm{KOAc}$ as base at $130{ }^{\circ} \mathrm{C}$ promotes 5 arylation of methyl 3-(pyrrol-1-yl)thiophene-2-carboxylate without decarboxylation; whereas, at $150{ }^{\circ} \mathrm{C}$ a $\mathrm{C} 5$ arylation followed by an in situ decarboxylation was observed. On the other hand, 1-(4-methylthiophen-3-yl)-pyrrole was selectively arylated at carbon $\mathrm{C} 2$ of the thienyl ring. From the decarboxylated 5-arylated thiophenes, the direct arylation at $\mathrm{C} 2$ position of thiophene was also found to be possible, and affords 2,5-diheteroarylated thiophenes bearing two different aryl units. A pyrazole bearing an ester substituent at $\mathrm{C} 4$ and a pyrrole substituent at $\mathrm{C} 5$ was only arylated at $\mathrm{C} 2$ on the pyrrole ring.

\section{Acknowledgments}

We thank the Centre National de la Recherche Scientifique, "Rennes Metropole", "UTIQUE" and Scientific Ministry of Higher Education Research of Tunisia for providing financial support.

\section{Experimental section}

\section{General Remarks}

All reactions were run under argon in Schlenk tubes using vacuum lines. DMA analytical grade was not distilled before use. KOAc (99\%) was used. Commercial heteroarenes and aryl bromides were used without purification. The reactions were followed by $\mathrm{GC}$ and NMR. ${ }^{1} \mathrm{H}$ and ${ }^{13} \mathrm{C}$ spectra were recorded with a Bruker 400 $\mathrm{MHz}$ spectrometer in $\mathrm{CDCl}_{3}$ solutions. Chemical shifts are reported in ppm relative to $\mathrm{CDCl}_{3}$ (7.25 for ${ }^{1} \mathrm{H} \mathrm{NMR}$ and 77.0 for ${ }^{13} \mathrm{C} \mathrm{NMR}$ ). Flash chromatography was performed on silica gel (230-400 mesh).

\section{General procedure}

In a typical experiment, the thiophene derivative (1.5 mmol), aryl bromide (1 mmol), KOAc (0.196 g, $2 \mathrm{mmol})$ and $\mathrm{Pd}(\mathrm{OAc})_{2}(2.24 \mathrm{mg}, 0.01 \mathrm{mmol})$ or $\mathrm{PdCl}\left(\mathrm{C}_{3} \mathrm{H}_{5}\right)(\mathrm{dppb})$ (12.1 $\mathrm{mg}, 0.02 \mathrm{mmol})$, were dissolved in DMA $(5 \mathrm{~mL})$ under an argon atmosphere. The reaction mixture was stirred at 130 or $150{ }^{\circ} \mathrm{C}$ for 2 or $17 \mathrm{~h}$ (see schemes). Then, the reaction was stirred at $30-40{ }^{\circ} \mathrm{C}$ in vacuum $(0.4$ mbar $)$ to remove the solvent, and the product was purified by silica gel column chromatography.

Preparation of the $\operatorname{PdCl}\left(\mathrm{C}_{3} \mathbf{H}_{5}\right)(\mathrm{dppb})$ catalyst: ${ }^{10} \mathrm{An}$ oven-dried $40 \mathrm{~mL}$ Schlenk tube equipped with a magnetic stirring bar under argon atmosphere, was charged with $\left[\mathrm{Pd}\left(\mathrm{C}_{3} \mathrm{H}_{5}\right) \mathrm{Cl}\right]_{2}(182 \mathrm{mg}, 0.5 \mathrm{mmol})$ and dppb (426 mg, 1 $\mathrm{mmol}) .10 \mathrm{~mL}$ of anhydrous dichloromethane were added, then, the solution was stirred at room temperature for twenty minutes. The solvent was removed in vacuum. The yellow powder was used without purification. ${ }^{31} \mathrm{P}$ NMR $\left(81 \mathrm{MHz}, \mathrm{CDCl}_{3}\right) \delta=19.3$ (s).

\section{Methyl 4-methyl-5-(3-nitrophenyl)-3-(pyrrol-1- yl)thiophene-2-carboxylate (1a)}

From methyl 4-methyl-3-(pyrrol-1-yl)thiophene-2carboxylate $(0.318 \mathrm{~g}, 1.5 \mathrm{mmol})$ and 3-bromonitrobenzene $(0.202 \mathrm{~g}, 1 \mathrm{mmol})$ at $130{ }^{\circ} \mathrm{C}$ during $2 \mathrm{~h}$ product 1a was obtained in $78 \%(0.267 \mathrm{~g})$ yield.

${ }^{1} \mathrm{H}$ NMR (400 MHz, $\mathrm{CDCl}_{3}$ ) $\delta 8.37(\mathrm{~s}, 1 \mathrm{H}), 8.29$ (d, $J=$ $8.0 \mathrm{~Hz}, 1 \mathrm{H}), 7.82(\mathrm{~d}, J=8.0 \mathrm{~Hz}, 1 \mathrm{H}), 7.67(\mathrm{t}, J=7.8 \mathrm{~Hz}$, $1 \mathrm{H}), 6.76(\mathrm{t}, J=2.0 \mathrm{~Hz}, 2 \mathrm{H}), 6.37(\mathrm{t}, J=2.0 \mathrm{~Hz}, 2 \mathrm{H}), 3.78$ (s, 3H), 2.07 (s, 3H).

${ }^{13} \mathrm{C} \mathrm{NMR}\left(100 \mathrm{MHz}, \mathrm{CDCl}_{3}\right) \delta 160.5,148.5,143.8,139.8$, $135.1,134.7,134.2,130.0,125.2,123.7,123.3,121.9$, $109.3,52.3,12.8$.

$\mathrm{C}_{17} \mathrm{H}_{14} \mathrm{~N}_{2} \mathrm{O}_{4} \mathrm{~S}$ (342.37): Calcd C 59.64, H 4.12; Found C 59.41, H 4.27.

\section{1-(4-Methyl-5-(3-nitrophenyl)thiophen-3-yl)-pyrrole} (1b)

From methyl 4-methyl-3-(pyrrol-1-yl)thiophene-2carboxylate $(0.318 \mathrm{~g}, 1.5 \mathrm{mmol})$ and 3-bromonitrobenzene $(0.202 \mathrm{~g}, 1 \mathrm{mmol})$ at $150{ }^{\circ} \mathrm{C}$ during $17 \mathrm{~h}$ product $\mathbf{1 b}$ was obtained in $76 \%(0.216 \mathrm{~g})$ yield.

${ }^{1} \mathrm{H}$ NMR (400 MHz, $\left.\mathrm{CDCl}_{3}\right) \delta 8.37(\mathrm{~s}, 1 \mathrm{H}), 8.25$ (d, $J=$ $8.0 \mathrm{~Hz}, 1 \mathrm{H}), 7.83(\mathrm{~d}, J=8.0 \mathrm{~Hz}, 1 \mathrm{H}), 7.65(\mathrm{t}, J=7.8 \mathrm{~Hz}$, $1 \mathrm{H}), 7.26(\mathrm{~s}, 1 \mathrm{H}), 6.88(\mathrm{t}, J=2.0 \mathrm{~Hz}, 2 \mathrm{H}), 6.36(\mathrm{t}, J=2.0$ $\mathrm{Hz}, 2 \mathrm{H}), 2.22$ (s, 3H). 
${ }^{13} \mathrm{C}$ NMR $\left(100 \mathrm{MHz}, \mathrm{CDCl}_{3}\right) \delta 148.5,141.0,136.3,134.7$, $135.7,130.8,129.7,123.7,122.4,121.9,117.9,109.3,13.1$. $\mathrm{C}_{15} \mathrm{H}_{12} \mathrm{~N}_{2} \mathrm{O}_{2} \mathrm{~S}$ (284.33): Calcd C 63.36, H 4.25; Found $\mathrm{C}$ $63.50, \mathrm{H} 4.37$.

\section{Methyl 5-(4-cyanophenyl)-4-methyl-3-(pyrrol-1- yl)thiophene-2-carboxylate (2)}

From methyl 4-methyl-3-(pyrrol-1-yl)thiophene-2carboxylate $(0.318 \mathrm{~g}, 1.5 \mathrm{mmol})$ and 4-bromobenzonitrile $(0.182 \mathrm{~g}, 1 \mathrm{mmol})$ at $130{ }^{\circ} \mathrm{C}$ during $2 \mathrm{~h}$ product 2 was obtained in $75 \%(0.241 \mathrm{~g})$ yield.

${ }^{1} \mathrm{H}$ NMR $\left(400 \mathrm{MHz}, \mathrm{CDCl}_{3}\right) \delta 7.76(\mathrm{~d}, J=8.0 \mathrm{~Hz}, 2 \mathrm{H})$, $7.61(\mathrm{~d}, J=8.0 \mathrm{~Hz}, 2 \mathrm{H}), 6.74(\mathrm{t}, J=2.0 \mathrm{~Hz}, 2 \mathrm{H}), 6.36(\mathrm{t}, J$ $=2.0 \mathrm{~Hz}, 2 \mathrm{H}), 3.78(\mathrm{~s}, 3 \mathrm{H}), 2.06(\mathrm{~s}, 3 \mathrm{H})$.

${ }^{13} \mathrm{C}$ NMR $\left(100 \mathrm{MHz}, \mathrm{CDCl}_{3}\right) \delta 141.1,139.2,136.4,132.5$, $130.8,129.3,121.9,118.6,118.2,111.2,109.3,13.3$.

$\mathrm{C}_{18} \mathrm{H}_{14} \mathrm{~N}_{2} \mathrm{O}_{2} \mathrm{~S}$ (322.38): Calcd C 67.06, H 4.38; Found $\mathrm{C}$ $67.24, \mathrm{H} 4.27$.

\section{Methyl 4-methyl-5-(4-propionylphenyl)-3-(pyrrol-1- yl)thiophene-2-carboxylate (3)}

From methyl 4-methyl-3-(pyrrol-1-yl)thiophene-2carboxylate $(0.318 \mathrm{~g}, \quad 1.5 \mathrm{mmol})$ and 4bromopropiophenone $(0.213 \mathrm{~g}, 1 \mathrm{mmol})$ at $130{ }^{\circ} \mathrm{C}$ during 2 $\mathrm{h}$ product 3 was obtained in $83 \%$ (0.293 g) yield.

${ }^{1} \mathrm{H}$ NMR (400 MHz, $\left.\mathrm{CDCl}_{3}\right) \delta 8.06(\mathrm{~d}, J=8.0 \mathrm{~Hz}, 2 \mathrm{H})$, $7.60(\mathrm{~d}, J=8.0 \mathrm{~Hz}, 2 \mathrm{H}), 6.75(\mathrm{t}, J=2.0 \mathrm{~Hz}, 2 \mathrm{H}), 6.36(\mathrm{t}, J$ $=2.0 \mathrm{~Hz}, 2 \mathrm{H}), 3.77(\mathrm{~s}, 3 \mathrm{H}), 3.04(\mathrm{q}, J=7.6 \mathrm{~Hz}, 2 \mathrm{H}), 2.06$ $(\mathrm{s}, 3 \mathrm{H}), 1.25(\mathrm{t}, J=7.6 \mathrm{~Hz}, 3 \mathrm{H})$.

${ }^{13} \mathrm{C}$ NMR $\left(100 \mathrm{MHz}, \mathrm{CDCl}_{3}\right) \delta 199.8,160.6,143.7,141.5$, $137.7,136.5,133.6,128.9,128.3,127.2,121.8,109.0,52.0$, $31.7,12.7,8.0$.

$\mathrm{C}_{20} \mathrm{H}_{19} \mathrm{NO}_{3} \mathrm{~S}$ (353.43): Calcd C 67.97, H 5.42; Found C 67.87, H 5.34.

\section{Methyl 5-(4-(methoxycarbonyl)phenyl)-4-methyl-3- (pyrrol-1-yl)thiophene-2-carboxylate (4) \\ From methyl 4-methyl-3-(pyrrol-1-yl)thiophene-2- carboxylate $(0.318 \mathrm{~g}, 1.5 \mathrm{mmol})$ and methyl 4- bromobenzoate $(0.215 \mathrm{~g}, 1 \mathrm{mmol})$ at $130{ }^{\circ} \mathrm{C}$ during $2 \mathrm{~h}$ product 3 was obtained in $82 \%(0.291 \mathrm{~g})$ yield. \\ ${ }^{1} \mathrm{H}$ NMR $\left(400 \mathrm{MHz}, \mathrm{CDCl}_{3}\right) \delta 8.13(\mathrm{~d}, J=8.0 \mathrm{~Hz}, 2 \mathrm{H})$, $7.58(\mathrm{~d}, J=8.0 \mathrm{~Hz}, 2 \mathrm{H}), 6.75(\mathrm{t}, J=2.0 \mathrm{~Hz}, 2 \mathrm{H}), 6.36(\mathrm{t}, J$ $=2.0 \mathrm{~Hz}, 2 \mathrm{H}), 3.95(\mathrm{~s}, 3 \mathrm{H}), 3.77(\mathrm{~s}, 3 \mathrm{H}), 2.06(\mathrm{~s}, 3 \mathrm{H})$. \\ ${ }^{13} \mathrm{C} \mathrm{NMR}\left(100 \mathrm{MHz}, \mathrm{CDCl}_{3}\right) \delta 166.4,160.7,143.9,141.7$, $137.9,133.7,130.1,128.9,127.2,122.0,109.3,109.2,52.3$, $52.1,12.8$ \\ $\mathrm{C}_{19} \mathrm{H}_{17} \mathrm{NO}_{4} \mathrm{~S}$ (355.41): Calcd C 64.21, H 4.82; Found $\mathrm{C}$ 64.02, H 4.99.}

\section{Methyl 5-(4-methoxyphenyl)-4-methyl-3-(pyrrol-1- yl)thiophene-2-carboxylate (5)}

From methyl 4-methyl-3-(pyrrol-1-yl)thiophene-2carboxylate $(0.318 \mathrm{~g}, 1.5 \mathrm{mmol})$ and 4-bromoanisole $(0.187$ $\mathrm{g}, 1 \mathrm{mmol})$ at $130{ }^{\circ} \mathrm{C}$ during $2 \mathrm{~h}$ product 5 was obtained in $78 \%(0.255 \mathrm{~g})$ yield.
${ }^{1} \mathrm{H}$ NMR (400 MHz, $\left.\mathrm{CDCl}_{3}\right) \delta 7.43(\mathrm{~d}, J=8.0 \mathrm{~Hz}, 2 \mathrm{H})$, $6.99(\mathrm{~d}, J=8.0 \mathrm{~Hz}, 2 \mathrm{H}), 6.75(\mathrm{t}, J=2.0 \mathrm{~Hz}, 2 \mathrm{H}), 6.35(\mathrm{t}, J$ $=2.0 \mathrm{~Hz}, 2 \mathrm{H}), 3.96(\mathrm{~s}, 3 \mathrm{H}), 3.76(\mathrm{~s}, 3 \mathrm{H}), 2.02(\mathrm{~s}, 3 \mathrm{H})$.

${ }^{13} \mathrm{C}$ NMR $\left(100 \mathrm{MHz}, \mathrm{CDCl}_{3}\right) \delta 161.2,160.2,144.0,143.5$, $132.3,130.4,126.0,123.2,122.1,114.5,109.1,55.5,52.2$, 12.9 .

$\mathrm{C}_{18} \mathrm{H}_{17} \mathrm{NO}_{3} \mathrm{~S}$ (327.40): Calcd $\mathrm{C}$ 66.03, $\mathrm{H}$ 5.23; Found $\mathrm{C}$ $65.89, \mathrm{H} 5.07$.

\section{Methyl 5-(3-acetylphenyl)-4-methyl-3-(pyrrol-1- yl)thiophene-2-carboxylate (6)}

From methyl 4-methyl-3-(pyrrol-1-yl)thiophene-2carboxylate $(0.318 \mathrm{~g}, 1.5 \mathrm{mmol})$ and 3-bromoacetophenone $(0.199 \mathrm{~g}, 1 \mathrm{mmol})$ at $130{ }^{\circ} \mathrm{C}$ during $2 \mathrm{~h}$ product 6 was obtained in $79 \%(0.268 \mathrm{~g})$ yield.

${ }^{1} \mathrm{H}$ NMR (400 MHz, $\left.\mathrm{CDCl}_{3}\right) \delta 8.08(\mathrm{~s}, 1 \mathrm{H}), 7.99(\mathrm{~d}, J=$ $8.0 \mathrm{~Hz}, 1 \mathrm{H}), 7.69(\mathrm{~d}, J=8.0 \mathrm{~Hz}, 1 \mathrm{H}), 7.58(\mathrm{t}, J=7.6 \mathrm{~Hz}$, $1 \mathrm{H}), 6.75(\mathrm{t}, J=2.0 \mathrm{~Hz}, 2 \mathrm{H}), 6.35(\mathrm{t}, J=2.0 \mathrm{~Hz}, 2 \mathrm{H}), 3.76$ (s, 3H), 2.65 (s, 3H), $2.04(\mathrm{~s}, 3 \mathrm{H})$.

${ }^{13} \mathrm{C} \mathrm{NMR}\left(100 \mathrm{MHz}, \mathrm{CDCl}_{3}\right) \delta 197.2,160.7,143.7,141.7$, $137.6,133.9,133.4,133.2,129.2,128.6,128.4,124.3$, 121.9, 109.0, 52.1, 26.6, 12.6 .

$\mathrm{C}_{19} \mathrm{H}_{17} \mathrm{NO}_{3} \mathrm{~S}$ (339.41): Calcd C 67.24, H 5.05; Found $\mathrm{C}$ $67.02, \mathrm{H} 5.30$.

Methyl 4-methyl-5-(4-nitro-3-(trifluoromethyl)phenyl)3-(pyrrol-1-yl)thiophene-2-carboxylate (7)

From methyl 4-methyl-3-(pyrrol-1-yl)thiophene-2carboxylate $(0.318 \mathrm{~g}, 1.5 \mathrm{mmol})$ and 4-bromo-1-nitro-2(trifluoromethyl)benzene $(0.270 \mathrm{~g}, 1 \mathrm{mmol})$ at $130{ }^{\circ} \mathrm{C}$ during $2 \mathrm{~h}$ product 7 was obtained in $60 \%(0.246 \mathrm{~g})$ yield.

${ }^{1} \mathrm{H}$ NMR $\left(400 \mathrm{MHz}, \mathrm{CDCl}_{3}\right) \delta 8.03(\mathrm{~d}, J=8.0 \mathrm{~Hz}, 1 \mathrm{H})$, $7.95(\mathrm{~s}, 1 \mathrm{H}), 7.84(\mathrm{~d}, J=8.0 \mathrm{~Hz}, 1 \mathrm{H}), 6.75(\mathrm{t}, J=2.0 \mathrm{~Hz}$, $2 \mathrm{H}), 6.38(\mathrm{t}, J=2.0 \mathrm{~Hz}, 2 \mathrm{H}), 3.79(\mathrm{~s}, 3 \mathrm{H}), 2.08(\mathrm{~s}, 3 \mathrm{H})$.

${ }^{13} \mathrm{C} \mathrm{NMR}\left(100 \mathrm{MHz}, \mathrm{CDCl}_{3}\right) \delta 160.3,144.0,138.5,138.1$, $135.2,133.0,128.2(\mathrm{q}, J=5.3 \mathrm{~Hz}), 126.4,125.9,124.5$ (q, $J=34.4 \mathrm{~Hz}), 121.8(\mathrm{q}, J=273.9 \mathrm{~Hz}), 121.9,109.5,52.4$, 12.9.

$\mathrm{C}_{18} \mathrm{H}_{13} \mathrm{~F}_{3} \mathrm{~N}_{2} \mathrm{O}_{4} \mathrm{~S}$ (410.37): Calcd C 52.68, H 3.19; Found C 52.74, H 3.04.

\section{Methyl 4-methyl-5-(pyridin-3-yl)-3-(pyrrol-1- yl)thiophene-2-carboxylate (8)}

From methyl 4-methyl-3-(pyrrol-1-yl)thiophene-2carboxylate $(0.318 \mathrm{~g}, 1.5 \mathrm{mmol})$ and 3-bromopyridine $(0.158 \mathrm{~g}, 1 \mathrm{mmol})$ at $130{ }^{\circ} \mathrm{C}$ during $2 \mathrm{~h}$ product 8 was obtained in $79 \%(0.235 \mathrm{~g})$ yield.

${ }^{1} \mathrm{H}$ NMR $\left(400 \mathrm{MHz}, \mathrm{CDCl}_{3}\right) \delta 8.77(\mathrm{~s}, 1 \mathrm{H}), 8.66(\mathrm{~d}, J=$ $4.2 \mathrm{~Hz}, 1 \mathrm{H}), 7.80(\mathrm{~d}, J=7.9 \mathrm{~Hz}, 1 \mathrm{H}), 7.41(\mathrm{dd}, J=7.9,4.2$ $\mathrm{Hz}, 1 \mathrm{H}), 6.75$ (s, 2H), $6.36(\mathrm{~s}, 2 \mathrm{H}), 3.77$ (s, 3H), 2.05 (s, $3 \mathrm{H})$.

${ }^{13} \mathrm{C} \mathrm{NMR}\left(100 \mathrm{MHz}, \mathrm{CDCl}_{3}\right) \delta 160.7,149.7,149.5,143.9$, $138.9,136.2$, 134.1, 129.7, 125.1, 123.6, 122.0, 109.2, 52.2, 12.7 .

$\mathrm{C}_{16} \mathrm{H}_{14} \mathrm{~N}_{2} \mathrm{O}_{2} \mathrm{~S}$ (298.36): Calcd C 64.41, H 4.73; Found C $64.55, \mathrm{H} 4.58$. 
Methyl 5-(5-cyanopyridin-3-yl)-4-methyl-3-(pyrrol-1yl)thiophene-2-carboxylate (9)

From methyl 4-methyl-3-(pyrrol-1-yl)thiophene-2carboxylate $(0.318 \mathrm{~g}, 1.5 \mathrm{mmol})$ and 5-bromonicotinonitrile $(0.183 \mathrm{~g}, 1 \mathrm{mmol})$ at $130{ }^{\circ} \mathrm{C}$ during $2 \mathrm{~h}$ product 9 was obtained in $58 \%(0.187 \mathrm{~g})$ yield.

${ }^{1} \mathrm{H}$ NMR (400 MHz, $\left.\mathrm{CDCl}_{3}\right) \delta 9.26(\mathrm{~s}, 1 \mathrm{H}), 8.89(\mathrm{~s}, 2 \mathrm{H})$, $6.74(\mathrm{t}, J=2.0 \mathrm{~Hz}, 2 \mathrm{H}), 6.37(\mathrm{t}, J=2.0 \mathrm{~Hz}, 2 \mathrm{H}), 3.79$ (s, $3 \mathrm{H}), 2.07$ (s, 3H).

${ }^{13} \mathrm{C}$ NMR $\left(100 \mathrm{MHz}, \mathrm{CDCl}_{3}\right) \delta 160.4,158.2,156.2,143.9$, $135.2,134.5,128.2,126.2,121.9,109.4,52.3,12.7$.

$\mathrm{C}_{17} \mathrm{H}_{13} \mathrm{~N}_{3} \mathrm{O}_{2} \mathrm{~S}$ (323.37): Calcd $\mathrm{C}$ 63.14, $\mathrm{H}$ 4.05; Found $\mathrm{C}$ 63.00, H 4.24.

\author{
Methyl 4-methyl-5-(pyrimidin-5-yl)-3-(pyrrol-1- \\ yl)thiophene-2-carboxylate (10) \\ From methyl 4-methyl-3-(pyrrol-1-yl)thiophene-2- \\ carboxylate $(0.318 \mathrm{~g}, 1.5 \mathrm{mmol})$ and 5-bromopyrimidine \\ $(0.159 \mathrm{~g}, 1 \mathrm{mmol})$ at $130{ }^{\circ} \mathrm{C}$ during $2 \mathrm{~h}$ product 10 was \\ obtained in $62 \%(0.185 \mathrm{~g})$ yield. \\ ${ }^{1} \mathrm{H}$ NMR (400 MHz, $\left.\mathrm{CDCl}_{3}\right) \delta 9.26(\mathrm{~s}, 1 \mathrm{H}), 8.86(\mathrm{~s}, 2 \mathrm{H})$, \\ $6.75(\mathrm{t}, J=2.0 \mathrm{~Hz}, 2 \mathrm{H}), 6.37(\mathrm{t}, J=2.0 \mathrm{~Hz}, 2 \mathrm{H}), 3.79(\mathrm{~s}$, \\ $3 \mathrm{H}), 2.07$ (s, 3H). \\ ${ }^{13} \mathrm{C} \mathrm{NMR}\left(100 \mathrm{MHz}, \mathrm{CDCl}_{3}\right) \delta 160.3,158.2,156.2,143.9$, \\ 135.2, 134.5, 128.2, 126.2, 121.9, 109.4, 52.4, 12.7 . \\ $\mathrm{C}_{15} \mathrm{H}_{13} \mathrm{~N}_{3} \mathrm{O}_{2} \mathrm{~S}$ (299.35): Calcd C 60.18, H 4.38; Found $\mathrm{C}$ \\ $60.01, \mathrm{H} 4.24$.
}

\section{4-(3-Methyl-4-(pyrrol-1-yl)thiophen-2-yl)benzonitrile}

(11)

From methyl 4-methyl-3-(pyrrol-1-yl)thiophene-2carboxylate $(0.318 \mathrm{~g}, 1.5 \mathrm{mmol})$ and 4-bromobenzonitrile $(0.182 \mathrm{~g}, 1 \mathrm{mmol})$ at $150{ }^{\circ} \mathrm{C}$ during $17 \mathrm{~h}$ product 11 was obtained in $90 \%$ ( $0.238 \mathrm{~g})$ yield.

${ }^{1} \mathrm{H}$ NMR $\left(400 \mathrm{MHz}, \mathrm{CDCl}_{3}\right) \delta 7.75(\mathrm{~d}, J=8.0 \mathrm{~Hz}, 2 \mathrm{H})$, $7.61(\mathrm{~d}, J=8.0 \mathrm{~Hz}, 2 \mathrm{H}), 7.26(\mathrm{~s}, 1 \mathrm{H}), 6.86(\mathrm{t}, J=2.0 \mathrm{~Hz}$, $2 \mathrm{H}), 6.34$ (t, $J=2.0 \mathrm{~Hz}, 2 \mathrm{H}), 2.20(\mathrm{~s}, 3 \mathrm{H})$.

${ }^{13} \mathrm{C}$ NMR $\left(100 \mathrm{MHz}, \mathrm{CDCl}_{3}\right) \delta 141.2,139.2,136.5,132.5$, $130.8,129.4,121.9,118.5,118.2,111.3,109.3,13.2$.

$\mathrm{C}_{16} \mathrm{H}_{12} \mathrm{~N}_{2} \mathrm{~S}$ (264.34): Calcd C 72.70, H 4.58; Found C $72.49, \mathrm{H} 4.37$.

\section{1-(4-(3-Methyl-4-(pyrrol-1-yl)thiophen-2-}

yl)phenyl)propan-1-one (12)

From methyl 4-methyl-3-(pyrrol-1-yl)thiophene-2carboxylate $(0.318 \mathrm{~g}, \quad 1.5 \mathrm{mmol})$ and 4bromopropiophenone $(0.213 \mathrm{~g}, 1 \mathrm{mmol})$ at $150{ }^{\circ} \mathrm{C}$ during $17 \mathrm{~h}$ product 12 was obtained in $72 \%(0.212 \mathrm{~g})$ yield.

${ }^{1} \mathrm{H}$ NMR $\left(400 \mathrm{MHz}, \mathrm{CDCl}_{3}\right) \delta 8.09(\mathrm{~d}, J=8.7 \mathrm{~Hz}, 2 \mathrm{H})$, $7.59(\mathrm{~d}, J=8.7 \mathrm{~Hz}, 2 \mathrm{H}), 7.21(\mathrm{~s}, 1 \mathrm{H}), 6.86(\mathrm{t}, J=2.0 \mathrm{~Hz}$, 2H), 6.33 (t, $J=2.0 \mathrm{~Hz}, 2 \mathrm{H}), 3.04(\mathrm{q}, \mathrm{J}=7.6 \mathrm{~Hz}, 2 \mathrm{H}), 2.20$ $(\mathrm{s}, 3 \mathrm{H}), 1.26(\mathrm{t}, J=7.6 \mathrm{~Hz}, 3 \mathrm{H})$.

${ }^{13} \mathrm{C}$ NMR $\left(100 \mathrm{MHz}, \mathrm{CDCl}_{3}\right) \delta 200.1,141.0,139.0,137.4$, $135.8,130.2,128.9,128.4,121.9,117.6,109.1,31.8,13.3$, 8.2 .

$\mathrm{C}_{18} \mathrm{H}_{17} \mathrm{NOS}$ (295.40): Calcd C 73.19, H 5.80; Found C 73.00, H 5.89.

\section{1-(5-(4-Chlorophenyl)-4-methylthiophen-3-yl)-pyrrole}

(13)

From methyl 4-methyl-3-(pyrrol-1-yl)thiophene-2carboxylate $(0.318 \mathrm{~g}, \quad 1.5 \mathrm{mmol})$ and 4bromochlorobenzene $(0.191 \mathrm{~g}, 1 \mathrm{mmol})$ at $150{ }^{\circ} \mathrm{C}$ during $17 \mathrm{~h}$ product 13 was obtained in $66 \%(0.180 \mathrm{~g})$ yield.

${ }^{1} \mathrm{H}$ NMR $\left(400 \mathrm{MHz}, \mathrm{CDCl}_{3}\right) \delta 7.42(\mathrm{~s}, 4 \mathrm{H}), 7.16(\mathrm{~s}, 1 \mathrm{H})$, $6.85(\mathrm{t}, J=2.0 \mathrm{~Hz}, 2 \mathrm{H}), 6.33(\mathrm{t}, J=2.0 \mathrm{~Hz}, 2 \mathrm{H}), 2.15$ (s, $3 \mathrm{H})$.

${ }^{13} \mathrm{C}$ NMR $\left(100 \mathrm{MHz}, \mathrm{CDCl}_{3}\right) \delta 140.7,137.3,133.8,133.0$, $130.2,129.5,128.9,121.9,116.8,109.1,13.1$.

$\mathrm{C}_{15} \mathrm{H}_{12} \mathrm{ClNS}$ (273.78): Calcd C 65.80, H 4.42; Found C $65.88, \mathrm{H} 4.28$.

\section{2-(3-Methyl-4-(pyrrol-1-yl)thiophen-2-yl)benzonitrile}

(15)

From methyl 4-methyl-3-(pyrrol-1-yl)thiophene-2carboxylate $(0.318 \mathrm{~g}, 1.5 \mathrm{mmol})$ and 2-bromobenzonitrile $(0.182 \mathrm{~g}, 1 \mathrm{mmol})$ at $150{ }^{\circ} \mathrm{C}$ during $17 \mathrm{~h}$ product 15 was obtained in $79 \%(0.208 \mathrm{~g})$ yield.

${ }^{1} \mathrm{H} \mathrm{NMR}\left(400 \mathrm{MHz}, \mathrm{CDCl}_{3}\right) \delta 7.82(\mathrm{~d}, J=8.2 \mathrm{~Hz}, 1 \mathrm{H})$, $7.70(\mathrm{t}, J=7.8 \mathrm{~Hz}, 1 \mathrm{H}), 7.60-7.47(\mathrm{~m}, 2 \mathrm{H}), 6.91(\mathrm{t}, J=2.0$ $\mathrm{Hz}, 2 \mathrm{H}), 6.35(\mathrm{t}, J=2.0 \mathrm{~Hz}, 2 \mathrm{H}), 2.12(\mathrm{~s}, 3 \mathrm{H})$.

${ }^{13} \mathrm{C}$ NMR $\left(100 \mathrm{MHz}, \mathrm{CDCl}_{3}\right) \delta 140.3,138.0,133.8,133.4$, $132.7,132.3,131.8,128.6,121.9,118.3,117.9,113.6$, 109.2, 13.2.

$\mathrm{C}_{16} \mathrm{H}_{12} \mathrm{~N}_{2} \mathrm{~S}$ (264.34): Calcd C 72.70, $\mathrm{H}$ 4.58; Found $\mathrm{C}$ 72.54, H 4.33 .

\section{1-(4-Methyl-5-(4-nitro-3-}

\section{(trifluoromethyl)phenyl)thiophen-3-yl)-pyrrole (16)}

From methyl 4-methyl-3-(pyrrol-1-yl)thiophene-2carboxylate $(0.318 \mathrm{~g}, 1.5 \mathrm{mmol})$ and 4-bromo-1-nitro-2(trifluoromethyl)benzene $(0.270 \mathrm{~g}, 1 \mathrm{mmol})$ at $150{ }^{\circ} \mathrm{C}$ during $17 \mathrm{~h}$ product 16 was obtained in $70 \%(0.246 \mathrm{~g})$ yield.

${ }^{1} \mathrm{H}$ NMR (400 MHz, $\left.\mathrm{CDCl}_{3}\right) \delta 8.01(\mathrm{~d}, J=8.0 \mathrm{~Hz}, 1 \mathrm{H})$, $7.93(\mathrm{~s}, 1 \mathrm{H}), 7.81(\mathrm{~d}, J=8.0 \mathrm{~Hz}, 1 \mathrm{H}), 7.31(\mathrm{~s}, 1 \mathrm{H}), 6.84(\mathrm{t}$, $J=2.0 \mathrm{~Hz}, 2 \mathrm{H}), 6.34(\mathrm{t}, J=2.0 \mathrm{~Hz}, 2 \mathrm{H}), 2.21(\mathrm{~s}, 3 \mathrm{H})$.

${ }^{13} \mathrm{C}$ NMR $\left(100 \mathrm{MHz}, \mathrm{CDCl}_{3}\right) \delta 146.6,141.4,139.7,134.5$, $132.6,131.9,128.0(\mathrm{q}, J=5.5 \mathrm{~Hz}), 125.8,124.2(\mathrm{q}, J=$ $34.0 \mathrm{~Hz}), 121.7$ (q, $J=272.5 \mathrm{~Hz}), 121.9,119.1,109.5$, 13.3 .

$\mathrm{C}_{16} \mathrm{H}_{11} \mathrm{~F}_{3} \mathrm{~N}_{2} \mathrm{O}_{2} \mathrm{~S}$ (352.33): Calcd C 54.54, H 3.15; Found C 54.47, H 3.07.

3-(3-Methyl-4-(pyrrol-1-yl)thiophen-2-yl)quinoline (17) From methyl 4-methyl-3-(pyrrol-1-yl)thiophene-2carboxylate $(0.318 \mathrm{~g}, 1.5 \mathrm{mmol})$ and 3-bromoquinoline $(0.208 \mathrm{~g}, 1 \mathrm{mmol})$ at $150{ }^{\circ} \mathrm{C}$ during $17 \mathrm{~h}$ product 17 was obtained in $61 \%(0.177 \mathrm{~g})$ yield.

${ }^{1} \mathrm{H}$ NMR (400 MHz, $\left.\mathrm{CDCl}_{3}\right) \delta 9.07(\mathrm{~s}, 1 \mathrm{H}), 8.22(\mathrm{~s}, 1 \mathrm{H})$, $8.16(\mathrm{~d}, J=8.0 \mathrm{~Hz}, 1 \mathrm{H}), 7.88(\mathrm{~d}, J=8.0 \mathrm{~Hz}, 1 \mathrm{H}), 7.75(\mathrm{t}, J$ $=7.8 \mathrm{~Hz}, 1 \mathrm{H}), 7.60(\mathrm{t}, J=7.8 \mathrm{~Hz}, 1 \mathrm{H}), 6.89$ (t, $J=2.0 \mathrm{~Hz}$, $2 \mathrm{H}), 6.35(\mathrm{t}, J=2.0 \mathrm{~Hz}, 2 \mathrm{H}), 2.24(\mathrm{~s}, 3 \mathrm{H})$. 
${ }^{13} \mathrm{C}$ NMR $\left(100 \mathrm{MHz}, \mathrm{CDCl}_{3}\right) \delta 150.5,147.2,141.0,135.2$, $134.7,130.6,129.8,129.3,127.9,127.8,127.6,127.2$, 121.8, 117.7, 109.2, 13.1.

$\mathrm{C}_{18} \mathrm{H}_{14} \mathrm{~N}_{2} \mathrm{~S}$ (290.38): Calcd C 74.45, H 4.86; Found C 74.67, H 5.01.

\section{5-(3-Methyl-4-(pyrrol-1-yl)thiophen-2-yl)nicotinonitrile} (18)

From methyl 4-methyl-3-(pyrrol-1-yl)thiophene-2carboxylate $(0.318 \mathrm{~g}, 1.5 \mathrm{mmol})$ and 5-bromonicotinonitrile $(0.183 \mathrm{~g}, 1 \mathrm{mmol})$ at $150{ }^{\circ} \mathrm{C}$ during $17 \mathrm{~h}$ product 18 was obtained in $76 \%(0.201 \mathrm{~g})$ yield.

${ }^{1} \mathrm{H}$ NMR $\left(400 \mathrm{MHz}, \mathrm{CDCl}_{3}\right) \delta 8.94(\mathrm{~s}, 1 \mathrm{H}), 8.87(\mathrm{~s}, 1 \mathrm{H})$, $8.04(\mathrm{~s}, 1 \mathrm{H}), 7.31(\mathrm{~s}, 1 \mathrm{H}), 6.85$ (t, $J=3.1 \mathrm{~Hz}, 2 \mathrm{H}), 6.34$ (t, $J$ $=3.1 \mathrm{~Hz}, 2 \mathrm{H}), 2.19(\mathrm{~s}, 3 \mathrm{H})$.

${ }^{13} \mathrm{C}$ NMR $\left(100 \mathrm{MHz}, \mathrm{CDCl}_{3}\right) \delta 152.6,150.8,141.2,138.7$, $131.9,131.8,131.2,121.9,119.0,116.1,109.5,13.1$.

$\mathrm{C}_{15} \mathrm{H}_{11} \mathrm{~N}_{3} \mathrm{~S}$ (265.33): Calcd C 67.90, H 4.18; Found $\mathrm{C}$ $68.06, \mathrm{H} 4.29$.

\section{5-(3-methyl-4-(pyrrol-1-yl)thiophen-2-yl)pyrimidine}

(19)

From methyl 4-methyl-3-(pyrrol-1-yl)thiophene-2carboxylate $(0.318 \mathrm{~g}, 1.5 \mathrm{mmol})$ and 5-bromopyrimidine $(0.159 \mathrm{~g}, 1 \mathrm{mmol})$ at $150{ }^{\circ} \mathrm{C}$ during $17 \mathrm{~h}$ product 19 was obtained in $74 \%(0.178 \mathrm{~g})$ yield.

${ }^{1} \mathrm{H}$ NMR (400 MHz, $\left.\mathrm{CDCl}_{3}\right) \delta 9.20(\mathrm{~s}, 1 \mathrm{H}), 8.87$ (s, 2H), $7.30(\mathrm{~s}, 1 \mathrm{H}), 6.85(\mathrm{t}, J=3.1 \mathrm{~Hz}, 2 \mathrm{H}), 6.34(\mathrm{t}, J=3.1 \mathrm{~Hz}$, $2 \mathrm{H}), 2.19(\mathrm{~s}, 3 \mathrm{H})$.

${ }^{13} \mathrm{C}$ NMR $\left(100 \mathrm{MHz}, \mathrm{CDCl}_{3}\right) \delta 157.6,156.3,141.3,131.9$, 130.7, 129.2, 122.0, 118.9, 109.6, 13.1 .

$\mathrm{C}_{13} \mathrm{H}_{11} \mathrm{~N}_{3} \mathrm{~S}$ (241.31): Calcd C 64.70, H 4.59; Found C 64.41, H 4.38.

Methyl 5-(4-nitrophenyl)-3-(pyrrol-1-yl)thiophene-2carboxylate (20a) and Methyl 3-(2-(4-nitrophenyl)pyrrol-1-yl)thiophene-2-carboxylate (20b)

From methyl 3-(pyrrol-1-yl)thiophene-2-carboxylate (0.310 g, $1.5 \mathrm{mmol})$ and 4-bromonitrobenzene $(0.202 \mathrm{~g}, 1 \mathrm{mmol})$ at $130{ }^{\circ} \mathrm{C}$ during $2 \mathrm{~h}$ a mixture of 20a and 20b was obtained in a 31:35 ratio. After column chromatography, roduct 20b was obtained in $35 \%(0.115 \mathrm{~g})$ yield, and product 20a was isolated in low yield.

20a: ${ }^{1} \mathrm{H} \mathrm{NMR}\left(400 \mathrm{MHz}, \mathrm{CDCl}_{3}\right) \delta 8.35(\mathrm{~d}, J=8.0 \mathrm{~Hz}$, $2 \mathrm{H}), 7.81(\mathrm{~d}, J=8.0 \mathrm{~Hz}, 2 \mathrm{H}), 7.44(\mathrm{~s}, 1 \mathrm{H}), 7.09-7.06(\mathrm{~m}$, $2 \mathrm{H}), 6.39-6.35$ (m, 2H), 3.89 (s, 3H).

20b: ${ }^{1} \mathrm{H}$ NMR $\left(400 \mathrm{MHz}, \mathrm{CDCl}_{3}\right) \delta 8.06(\mathrm{~d}, J=8.0 \mathrm{~Hz}$, 2H), $7.52(\mathrm{~d}, J=5.2 \mathrm{~Hz}, 1 \mathrm{H}), 7.22(\mathrm{~d}, J=8.0 \mathrm{~Hz}, 2 \mathrm{H}), 6.97$ $(\mathrm{t}, J=1.3 \mathrm{~Hz}, 1 \mathrm{H}), 6.91(\mathrm{~d}, J=5.2 \mathrm{~Hz}, 1 \mathrm{H}), 6.64(\mathrm{dd}, J=$ $3.6,1.6 \mathrm{~Hz}, 1 \mathrm{H}), 6.43(\mathrm{t}, J=3.3 \mathrm{~Hz}, 1 \mathrm{H}), 3.71(\mathrm{~s}, 3 \mathrm{H})$.

${ }^{13} \mathrm{C}$ NMR $\left(100 \mathrm{MHz}, \mathrm{CDCl}_{3}\right) \delta 160.4,145.7,142.1,139.1$, $132.9,130.6,128.4,127.1,127.0,125.0,123.6,112.5$, $110.2,52.2$

$\mathrm{C}_{16} \mathrm{H}_{12} \mathrm{~N}_{2} \mathrm{O}_{4} \mathrm{~S}$ (328.34): Calcd C 58.53, H 3.68; Found C 59.01, H 3.55.

\section{4-(4-Methyl-3-(pyrrol-1-yl)thiophen-2-yl)benzonitrile} (21)

From 1-(4-methylthiophen-3-yl)-pyrrole $(0.326 \mathrm{~g}, 2 \mathrm{mmol})$ and 4-bromobenzonitrile $(0.182 \mathrm{~g}, 1 \mathrm{mmol})$ at $130{ }^{\circ} \mathrm{C}$ during $2 \mathrm{~h}$ product 21 was obtained in $46 \%(0.121 \mathrm{~g})$ yield.

${ }^{1} \mathrm{H} \mathrm{NMR}\left(400 \mathrm{MHz}, \mathrm{CDCl}_{3}\right) \delta 7.50(\mathrm{~d}, J=8.0 \mathrm{~Hz}, 2 \mathrm{H})$, 7.07 (s, 1H), $7.02(\mathrm{~d}, J=8.0 \mathrm{~Hz}, 2 \mathrm{H}), 6.59$ (t, $J=2.0 \mathrm{~Hz}$, $2 \mathrm{H}), 6.33(\mathrm{t}, J=2.0 \mathrm{~Hz}, 2 \mathrm{H}), 2.08(\mathrm{~s}, 3 \mathrm{H})$.

${ }^{13} \mathrm{C}$ NMR $\left(100 \mathrm{MHz}, \mathrm{CDCl}_{3}\right) \delta 136.8,136.7,136.6,134.8$, 132.4, 127.2, 121.3, 120.8, 118.6, 111.0, 110.1, 13.6.

$\mathrm{C}_{16} \mathrm{H}_{12} \mathrm{~N}_{2} \mathrm{~S}$ (264.34): Calcd C 72.70, $\mathrm{H}$ 4.58; Found C $72.78, \mathrm{H} 4.79$.

\section{3-(3-Methyl-5-phenyl-4-(pyrrol-1-yl)thiophen-2- yl)quinoline (22)}

From 3-(3-methyl-4-(pyrrol-1-yl)thiophen-2-yl)quinoline 17 (0.435 g, $1.5 \mathrm{mmol})$ and bromobenzene $(0.157 \mathrm{~g}, 1$ $\mathrm{mmol}$ ) at $150{ }^{\circ} \mathrm{C}$ during $17 \mathrm{~h}$ product 22 was obtained in $56 \%(0.205 \mathrm{~g})$ yield.

${ }^{1} \mathrm{H}$ NMR (400 MHz, $\left.\mathrm{CDCl}_{3}\right) \delta 9.11(\mathrm{~s}, 1 \mathrm{H}), 8.27(\mathrm{~s}, 1 \mathrm{H})$, $8.15(\mathrm{~d}, J=8.0 \mathrm{~Hz}, 1 \mathrm{H}), 7.89(\mathrm{~d}, J=8.0 \mathrm{~Hz}, 1 \mathrm{H}), 7.76(\mathrm{t}, J$ $=7.8 \mathrm{~Hz}, 1 \mathrm{H}), 7.61(\mathrm{t}, J=7.8 \mathrm{~Hz}, 1 \mathrm{H}), 7.30-7.24(\mathrm{~m}, 3 \mathrm{H})$, $7.07(\mathrm{~d}, J=8.0 \mathrm{~Hz}, 2 \mathrm{H}), 6.71(\mathrm{t}, J=2.0 \mathrm{~Hz}, 2 \mathrm{H}), 6.33(\mathrm{t}, J$ $=2.0 \mathrm{~Hz}, 2 \mathrm{H}), 2.17(\mathrm{~s}, 3 \mathrm{H})$.

${ }^{13} \mathrm{C}$ NMR $\left(100 \mathrm{MHz}, \mathrm{CDCl}_{3}\right) \delta 150.5,137.5,135.8,135.0$, $133.7,131.8,129.9,129.4,128.8,128.1,127.9,127.7$, 127.3, 127.1, 121.7, 109.7, 12.9 .

$\mathrm{C}_{24} \mathrm{H}_{18} \mathrm{~N}_{2} \mathrm{~S}$ (366.48): Calcd C 78.66, $\mathrm{H}$ 4.95; Found $\mathrm{C}$ 78.54, H 5.11.

\section{1-(4-(3-Methyl-5-(pyrimidin-5-yl)-4-(pyrrol-1-}

yl)thiophen-2-yl)phenyl)propan-1-one (23)

From methyl 4-methyl-5-(4-propionylphenyl)-3-(pyrrol-1yl)thiophene-2-carboxylate $3(0.529 \mathrm{~g}, 1.5 \mathrm{mmol}) 5$ bromopyrimidine $(0.159 \mathrm{~g}, 1 \mathrm{mmol})$ at $150{ }^{\circ} \mathrm{C}$ during $17 \mathrm{~h}$ product 23 was obtained in $71 \%(0.264 \mathrm{~g})$ yield.

${ }^{1} \mathrm{H}$ NMR (400 MHz, $\left.\mathrm{CDCl}_{3}\right) \delta 9.05(\mathrm{~s}, 1 \mathrm{H}), 8.26(\mathrm{~s}, 2 \mathrm{H})$, $8.08(\mathrm{~d}, J=8.7 \mathrm{~Hz}, 2 \mathrm{H}), 7.63(\mathrm{~d}, J=8.7 \mathrm{~Hz}, 2 \mathrm{H}), 6.68(\mathrm{t}, J$ $=2.0 \mathrm{~Hz}, 2 \mathrm{H}), 6.39(\mathrm{t}, J=2.0 \mathrm{~Hz}, 2 \mathrm{H}), 3.05(\mathrm{q}, \mathrm{J}=7.6 \mathrm{~Hz}$, $2 \mathrm{H}), 2.16(\mathrm{~s}, 3 \mathrm{H}), 1.27(\mathrm{t}, J=7.6 \mathrm{~Hz}, 3 \mathrm{H})$.

${ }^{13} \mathrm{C}$ NMR $\left(100 \mathrm{MHz}, \mathrm{CDCl}_{3}\right) \delta 200.0,157.4,154.2,138.0$, $137.1,136.3,133.5,129.3,128.8,128.6,126.8,121.2$, $110.9,31.9,12.9,8.2$.

$\mathrm{C}_{22} \mathrm{H}_{19} \mathrm{~N}_{3} \mathrm{OS}$ (373.47): Calcd C 70.75, H 5.13; Found $\mathrm{C}$ 70.82 , H 5.01 .

\section{5-(3-Nitrophenyl)-2-(pyrrol-1-yl)thiophene-3- carbonitrile (24a)}

From 2-(pyrrol-1-yl)thiophene-3-carbonitrile (0.261 g, 1.5 $\mathrm{mmol})$ and 3-bromonitrobenzene $(0.202 \mathrm{~g}, 1 \mathrm{mmol})$ at 150 ${ }^{\circ} \mathrm{C}$ during $17 \mathrm{~h}$ product $24 \mathrm{a}$ was obtained in $<10 \%(0.029$ g) yield. This compound was not isolated in pure form.

${ }^{1} \mathrm{H}$ NMR (400 MHz, $\left.\mathrm{CDCl}_{3}\right) \delta 8.38(\mathrm{~s}, 1 \mathrm{H}), 8.29(\mathrm{~d}, J=$ $8.0 \mathrm{~Hz}, 1 \mathrm{H}), 7.84(\mathrm{~d}, J=8.0 \mathrm{~Hz}, 1 \mathrm{H}), 7.63(\mathrm{t}, J=7.8 \mathrm{~Hz}$, $1 \mathrm{H}), 7.27$ (t, $J=2.0 \mathrm{~Hz}, 2 \mathrm{H}), 6.44$ (t, $J=2.0 \mathrm{~Hz}, 2 \mathrm{H})$.

\section{Ethyl 5-(2-(4-cyanophenyl)-pyrrol-1-yl)-1-} methylpyrazole-4-carboxylate $(25 b)$ 
From ethyl 1-methyl-5-(pyrrol-1-yl)-pyrazole-4carboxylate $(0.328 \mathrm{~g}, 1.5 \mathrm{mmol})$ and 4-bromobenzonitrile $(0.182 \mathrm{~g}, 1 \mathrm{mmol})$ at $130{ }^{\circ} \mathrm{C}$ during $2 \mathrm{~h}$ product $25 \mathbf{b}$ was obtained in $42 \%(0.134 \mathrm{~g})$ yield.

${ }^{1} \mathrm{H}$ NMR (400 MHz, $\left.\mathrm{CDCl}_{3}\right) \delta 7.98(\mathrm{~s}, 1 \mathrm{H}), 7.50(\mathrm{~d}, J=$ $8.0 \mathrm{~Hz}, 2 \mathrm{H}), 7.14(\mathrm{~d}, J=8.0 \mathrm{~Hz}, 2 \mathrm{H}), 6.88-6.80(\mathrm{~m}, 1 \mathrm{H})$, 6.69-6.66 (m, 1H), $6.49(\mathrm{t}, J=3.1 \mathrm{~Hz}, 1 \mathrm{H}), 4.14(\mathrm{q}, J=7.6$ $\mathrm{Hz}, 2 \mathrm{H}), 3.40$ (s, 3H), 1.17 (t, $J=7.6 \mathrm{~Hz}, 3 \mathrm{H})$.

${ }^{13} \mathrm{C}$ NMR $\left(100 \mathrm{MHz}, \mathrm{CDCl}_{3}\right) \delta 161.3,141.1,139.6,136.1$, $133.3,132.5,126.4,126.2,118.6,112.4,111.7,110.2$, $110,1,60.4,35.7,14.1$.

$\mathrm{C}_{18} \mathrm{H}_{16} \mathrm{~N}_{4} \mathrm{O}_{2}$ (320.35): Calcd $\mathrm{C}$ 67.49, H 5.03; Found $\mathrm{C}$ 67.66, H 4.78.

\section{Ethyl 1-methyl-5-(2-(4-nitrophenyl)-pyrrol-1-yl)- pyrazole-4-carboxylate (26)}

From ethyl 1-methyl-5-(pyrrol-1-yl)-pyrazole-4carboxylate $(0.328 \mathrm{~g}, 1.5 \mathrm{mmol})$ and 4-bromonitrobenzene $(0.202 \mathrm{~g}, 1 \mathrm{mmol})$ at $130{ }^{\circ} \mathrm{C}$ during $2 \mathrm{~h}$ product 26 was obtained in $40 \%(0.136 \mathrm{~g})$ yield.

${ }^{1} \mathrm{H} \mathrm{NMR}\left(400 \mathrm{MHz}, \mathrm{CDCl}_{3}\right) \delta 8.13(\mathrm{~d}, J=8.0 \mathrm{~Hz}, 2 \mathrm{H})$, $8.02(\mathrm{~s}, 1 \mathrm{H}), 7.21(\mathrm{~d}, J=8.0 \mathrm{~Hz}, 2 \mathrm{H}), 6.87(\mathrm{t}, J=3.1 \mathrm{~Hz}$, $1 \mathrm{H}), 6.79-6.75(\mathrm{~m}, 1 \mathrm{H}), 6.54(\mathrm{t}, J=3.1 \mathrm{~Hz}, 1 \mathrm{H}), 4.18(\mathrm{q}, J$ $=7.6 \mathrm{~Hz}, 2 \mathrm{H}), 3.46(\mathrm{~s}, 3 \mathrm{H}), 1.20(\mathrm{t}, J=7.6 \mathrm{~Hz}, 3 \mathrm{H})$.

${ }^{13} \mathrm{C}$ NMR $\left(100 \mathrm{MHz}, \mathrm{CDCl}_{3}\right) \delta 161.3,146.1,141.1,138.0$, 133.1, 126.6, 126.3, 124.1, 113.0, 111.9, 110.2, 60.4, 35.7, 14.1.

$\mathrm{C}_{17} \mathrm{H}_{16} \mathrm{~N}_{4} \mathrm{O}_{4}$ (340.33): Calcd C 59.99, H 4.74; Found C $60.11, \mathrm{H} 4.59$.

\section{Ethyl 5-(2-(4-formylphenyl)-pyrrol-1-yl)-1- methylpyrazole-4-carboxylate (27) \\ From ethyl 1-methyl-5-(pyrrol-1-yl)-pyrazole-4-} carboxylate $(0.328 \mathrm{~g}, 1.5 \mathrm{mmol})$ and 4-bromobenzaldehyde $(0.185 \mathrm{~g}, 1 \mathrm{mmol})$ at $130{ }^{\circ} \mathrm{C}$ during $2 \mathrm{~h}$ product 27 was obtained in $50 \%(0.161 \mathrm{~g})$ yield.

${ }^{1} \mathrm{H}$ NMR (400 MHz, CDCl $\left.{ }_{3}\right) \delta 9.98(\mathrm{~s}, 1 \mathrm{H}), 7.99(\mathrm{~s}, 1 \mathrm{H})$, $7.74(\mathrm{~d}, J=8.0 \mathrm{~Hz}, 2 \mathrm{H}), 7.21(\mathrm{~d}, J=8.0 \mathrm{~Hz}, 2 \mathrm{H}), 6.87(\mathrm{t}, J$ $=3.1 \mathrm{~Hz}, 1 \mathrm{H}), 6.74-6.70(\mathrm{~m}, 1 \mathrm{H}), 6.50(\mathrm{t}, J=3.1 \mathrm{~Hz}, 1 \mathrm{H})$, $4.16(\mathrm{q}, J=7.6 \mathrm{~Hz}, 2 \mathrm{H}), 3.40$ (s, 3H), $1.17(\mathrm{t}, J=7.6 \mathrm{~Hz}$, $3 \mathrm{H})$.

${ }^{13} \mathrm{C}$ NMR $\left(100 \mathrm{MHz}, \mathrm{CDCl}_{3}\right) \delta 191.4,161.4,141.1,139.8$, $137.5,134.5,133.9,130.2,126.4,126.2,112.3,111.6$, $110.1,60.3,35.7,14.1$.

$\mathrm{C}_{18} \mathrm{H}_{17} \mathrm{~N}_{3} \mathrm{O}_{3}$ (323.34): Calcd $\mathrm{C}$ 66.86, H 5.30; Found $\mathrm{C}$ 67.04, H 5.07.

\section{Ethyl 1-methyl-5-(2-(4-nitro-3-(trifluoromethyl)phenyl)- pyrrol-1-yl)-pyrazole-4-carboxylate (28)}

From ethyl 1-methyl-5-(pyrrol-1-yl)-pyrazole-4carboxylate $(0.328 \mathrm{~g}, 1.5 \mathrm{mmol})$ and 4-bromo-1-nitro-2(trifluoromethyl)benzene $(0.270 \mathrm{~g}, 1 \mathrm{mmol})$ at $130{ }^{\circ} \mathrm{C}$ during $2 \mathrm{~h}$ product 28 was obtained in $56 \%(0.228 \mathrm{~g})$ yield. ${ }^{1} \mathrm{H}$ NMR $\left(400 \mathrm{MHz}, \mathrm{CDCl}_{3}\right) \delta 7.99(\mathrm{~s}, 1 \mathrm{H}), 7.80(\mathrm{~d}, J=$ $8.0 \mathrm{~Hz}, 1 \mathrm{H}), 7.59(\mathrm{~s}, 1 \mathrm{H}), 7.25(\mathrm{~d}, J=8.0 \mathrm{~Hz}, 1 \mathrm{H}), 6.90(\mathrm{t}$, $J=3.1 \mathrm{~Hz}, 1 \mathrm{H}), 6.80-6.75(\mathrm{~m}, 1 \mathrm{H}), 6.54(\mathrm{t}, J=3.1 \mathrm{~Hz}$, $1 \mathrm{H}), 4.16(\mathrm{q}, J=7.6 \mathrm{~Hz}, 2 \mathrm{H}), 3.48(\mathrm{~s}, 3 \mathrm{H}), 1.18(\mathrm{t}, J=7.6$ $\mathrm{Hz}, 3 \mathrm{H})$.
${ }^{13} \mathrm{C}$ NMR $\left(100 \mathrm{MHz}, \mathrm{CDCl}_{3}\right) \delta 161.1,141.1,139.0,136.6$, $131.9,129.0,127.1,126.0,125.4(\mathrm{q}, J=5.6 \mathrm{~Hz}), 124.5$ (q, $J=34.0 \mathrm{~Hz}), 121.6(\mathrm{q}, J=273.7 \mathrm{~Hz}), 113.6,112.2,110.5$, $60.5,35.7,14.0$.

$\mathrm{C}_{18} \mathrm{H}_{15} \mathrm{~F}_{3} \mathrm{~N}_{4} \mathrm{O}_{4}$ (408.33): Calcd C 52.95, H 3.70; Found C $52.79, \mathrm{H} 3.89$.

\section{Ethyl 1-methyl-5-(2-(pyrimidin-5-yl)-pyrrol-1-yl)- pyrazole-4-carboxylate (29)}

From ethyl 1-methyl-5-(pyrrol-1-yl)-pyrazole-4carboxylate $(0.328 \mathrm{~g}, 1.5 \mathrm{mmol})$ and 5-bromopyrimidine $(0.159 \mathrm{~g}, 1 \mathrm{mmol})$ at $130{ }^{\circ} \mathrm{C}$ during $2 \mathrm{~h}$ product 29 was obtained in $58 \%(0.172 \mathrm{~g})$ yield.

${ }^{1} \mathrm{H}$ NMR (400 MHz, $\left.\mathrm{CDCl}_{3}\right) \delta 9.02(\mathrm{~s}, 1 \mathrm{H}), 8.45(\mathrm{~s}, 2 \mathrm{H})$, $7.96(\mathrm{~s}, 1 \mathrm{H}), 6.87(\mathrm{t}, J=3.1 \mathrm{~Hz}, 1 \mathrm{H}), 6.75-6.70(\mathrm{~m}, 1 \mathrm{H})$, $6.53(\mathrm{t}, J=3.1 \mathrm{~Hz}, 1 \mathrm{H}), 4.13(\mathrm{q}, J=7.6 \mathrm{~Hz}, 2 \mathrm{H}), 3.48(\mathrm{~s}$, $3 \mathrm{H}), 1.18$ (t, $J=7.6 \mathrm{~Hz}, 3 \mathrm{H})$.

${ }^{13} \mathrm{C}$ NMR $\left(100 \mathrm{MHz}, \mathrm{CDCl}_{3}\right) \delta 160.8,156.5,153.7,140.8$, $138.7,128.3,125.9,125.7,111.9,111.7,110.2,60.1,35.3$, 13.7.

$\mathrm{C}_{15} \mathrm{H}_{15} \mathrm{~N}_{5} \mathrm{O}_{2}$ (297.31): Calcd C 60.60, H 5.09; Found C $60.41, \mathrm{H} 5.27$.

\section{References}

1. (a) Schulz, V.; Fischer, W.; Hanselle, U.; Huhmann, W.; Zietsch, V. Eur. J. Clin. Pharmacol. 1986, 31, 411-414; (b) Herling, A. W.; Scholl, T.; Bickel, M.; Lang, H. J.; Scheunemann, K. H.; Weidmann, K.; Rippel, R. Pharmacology 1991, 43, 293-303; (c) Maj, J.; Rogoz, Z.; Sowinska, H.; Zalewski, Z. Pol. J. Pharmacol. Pharm. 1987, 39, 63-74.

2. (a) Akita, Y.; Inoue, A.; Yamamoto, K.; Ohta, A.; Kurihara, T.; Shimizu, M. Heterocycles 1985, 23, 2327-2333.; (b) Ohta, A.; Akita, Y.; Ohkuwa, T.; Chiba, M.; Fukunaga, R.; Miyafuji, A.; Nakata, T.; Tani, N.; Aoyagi, Y. Heterocycles 1990, 31, 1951-1958; (c) Aoyagi, Y.; Inoue, A.; Koizumi, I.; Hashimoto, R.; Miyafuji, A.; Kunoh, J.; Honma, R. Akita, Y.; Ohta, A. Heterocycles 1992, 33, 257-272.

3. For reviews on $\mathrm{Pd}$-catalysed $\mathrm{C}-\mathrm{H}$ functionalisations: (a) Alberico, D.; Scott, M. E.; Lautens, M. Chem. Rev. 2007, 107, 174-238; (b) Satoh, T.; Miura, M. Chem. Lett. 2007, 36, 200-205; (c) Li, B.-J.; Yang, S.-D.; Shi, Z.-J. Synlett 2008, 949-957; (d) Bellina, F.; Rossi, R. Tetrahedron 2009, 65, 10269-10310; (e) Li, B.-J.; Yang, S.-D.; Shi, Z.-J. Synlett 2008, 949-957; (f) Ackermann, L.; Vincente, R.; Kapdi, A. R. Angew. Chem. Int. Ed. 2009, 48, 9792-9826; (g) Roger, J.; Gottumukkala, A. L.; Doucet, H. ChemCatChem 2010, 2, 2040; (h) Wu, X.-F.; Anbarasan, P.; Neumann, H.; Beller M. Angew. Chem. Int. Ed. 2010, 49, 7316-7319; (i) Kuhl, N.; Hopkinson, M. N.; Wencel-Delord, J.; Glorius, F. Angew. Chem. Int. Ed. 2012, 51, 10236-10254; (j) Yamaguchi, J.; Yamaguchi, A. D.; Itami, K. Angew. Chem., Int. Ed. 2012, 51, 8960-9009; (k) Wencel-Delord, J.; Glorius, F. Nature Chem. 2013, 5, 369-375; (1) Kuzhushkov, S. I.; Potukuchi, H. K.; Ackermann, L. Catal. Sci. Technol. 2013, 3, 562-571; (m) Rossi, R.; Bellina, F.; Lessi, M.; Manzini, C. Adv. Synth. Catal. 2014, 356, 17-117; (n) He, M.; Soulé, J. F.; Doucet, H. ChemCatChem 2014, 6, 1824-1859; (o) Yuan, K.; Soulé, J. F.; Doucet, H. ACS Catal. 2015, 5, 978-991.

4. For selected examples of Pd-catalysed direct arylations from our laboratory: (a) Beydoun, K.; Zaarour, M.; Williams, J. A. G.; Doucet, H.; Guerchais V. Chem. Commun. 2012, 48, 
1260-1262; (b) Fu, H. Y.; Chen, L. Doucet, H. J. Org. Chem. 2012, 77, 4473-4478; (c) Zhao, L.; Bruneau, C.; Doucet, H. Chem. Commun. 2013, 49, 5598-5600.

5. (a) Li, J. J.; Gribble, G. W. Palladium in Heterocyclic Chemistry, Pergamon: Amsterdam, 2000; (b) Negishi, E. Ed. Handbook of Organopalladium Chemistry for Organic Synthesis; Wiley-Interscience: New York, 2002; Part III, p 213; (c) Schnürch, M.; Flasik, R.; Khan, A. F.; Spina M.; Mihovilovic, M. D.; Stanetty, P. Eur. J. Org. Chem. 2006, 3283-3307; (d) Ackermann, L. Modern arylation methods, Wiley, 2009.

6. For selected examples of Pd-catalysed direct arylations of thiophene derivatives: (a) Masui, K.; Ikegami H.; Mori, A. $J$. Am. Chem. Soc., 2004, 126, 5074-5075; (b) David, E.; PelletRostaing, S.; Lemaire, M. Tetrahedron 2007, 63, 8999-9006; (c) Amaladass, P.; Clement, J. A.; Mohanakrishnan, A. K. Tetrahedron 2007, 63, 10363-10371; (d) Nakano, M.; Tsurugi, H.; Satoh, T.; Miura, M. Org. Lett. 2008, 10, 18511854; (e) Liégault, B.; Petrov, I.; Gorlesky, S. I.; Fagnou, K. J. Org. Chem. 2010, 75, 1047-1060; (f) Chen, L.; Roger, J.; Bruneau, C.; Dixneuf, P. H.; Doucet, H. Chem. Commun. 2011, 47, 1872-1874; (g) Yuan, K.; Doucet, H. Chem. Sci. 2014, 5, 392-396.

7. For selected examples of Pd-catalysed direct arylations of pyrrole derivatives: (a) Toure, B. B.; Lane, B. S.; Sames, D. Org. Lett. 2006, 8, 1979-1982; (b) Yang, S.-D.; Sun, C.-L.; Fang, Z.; Li, B.-J.; Li, Y.-Z.; Shi, Z.-J. Angew. Chem., Int. Ed. 2008, 47, 1473-1476; (c) Liégaut, B.; Lapointe, D.; Caron, L.; Vlassova, A.; Fagnou, K. J. Org. Chem. 2009, 74, 1826-1834; (d) Roger, J.; Doucet, H. Adv. Synth. Catal. 2009, 351, 1977-1990; (e) Gryko, D. T.; Vakuliuk, O.; Gryko, D.; Koszarna, B. J. Org. Chem. 2009, 74, 9517-9520; (f) Nadres, E. T.; Lazareva, A.; Daugulis, O. J. Org. Chem. 2011, 76, 471-483; (g) Vakuliuk, O.; Koszarna, B.; Gryko, D. T. Adv. Synth. Catal. 2011, 353, 925-930; (h) Wu, M.; Luo, J.; Xiao, F.; Zhang, S.; Deng, G.-J.; Luo, H.-A. Adv. Synth. Catal. 2012, 354, 335-340; (i) Florentino, L.; Aznar, F.; Valdes, C. Chem. Eur. J. 2013, 19, 10506-10510; (j) Ehlers, P.; Petrosyan, A.; Baumgard, J.; Jopp, S.; Steinfeld, N.; Ghochikyan, T. V.; Saghyan, A. S.; Fischer, C.; Langer, P. ChemCatChem 2013, 5, 2504-2511; (k) Zhao, L.; Bruneau, C.; Doucet H. ChemCatChem 2013, 5, 255-262; (1) Jin, R.; Yuan, K.; Chatelain, E.; Soulé, J.-F.; Doucet H. Adv. Synth. Catal. 2014, 356, 3831-3841.

8. (a) Roger, J.; Požgan, F.; Doucet, H. Green Chem. 2009, 11, 425-432; (b) Xu, Y.; Zhao, L.; Li, Y.; Doucet, H. Adv. Synth. Catal. 2013, 355, 1423-1432.

9. Derridj, F.; Roger, J.; Djebbar, S.; Doucet, H. Org. Lett. 2010 , $12,4320-4323$.

10. Cantat, T.; Génin, E.; Giroud, C.; Meyer G.; Jutand, A. J. Organomet. Chem. 2003, 687, 365-376.

Keywords: palladium, catalysis, $\mathrm{C}-\mathrm{H}$ bond activation, thiophenes pyrroles, direct arylation, aryl halides 